\title{
A family of LIM domain-associated cofactors confer transcriptional synergism between LIM and Otx homeodomain proteins
}

\author{
Ingolf Bach, ${ }^{1}$ Catherine Carrière, Heather P. Ostendorff, ${ }^{3}$ Bogi Andersen ${ }^{2}$ and Michael G. Rosenfeld ${ }^{1}$ \\ Howard Hughes Medical Institute, Eukaryotic Regulatory Biology Program, ${ }^{2}$ Division of Endocrinology and Metabolism, \\ University of California, San Diego, School of Medicine, La Jolla, California 92093-0648 USA; ${ }^{3}$ The Salk Institute for \\ Biological Sciences, San Diego, California 92186-5800
}

The essential roles of LIM homeodomain proteins in cell fate determination during development have been demonstrated in organisms as divergent as Drosophila and higher mammals. We have isolated murine cDNAs encoding two highly homologous proteins that specifically interact with the LIM domains of P-Lim/Lhx3 and several other LIM homeodomain factors. Transcripts encoding these factors can be detected as early as mouse E8.5, with maximal expression observed in regions of the embryo in which the LIM homeodomain factors P-Lim/Lhx3, Isl-1, and LH-2 are selectively expressed. These proteins can potentiate transactivation by P-Lim/Lhx-3 and are required for a synergistic activation of the glycoprotein hormone $\alpha$-subunit promoter by P-Lim/Lhx3 and a pituitary Otx class homeodomain transcription factor, with which they also specifically associate. Our results link LIM homeodomain proteins and members of the Otx class of transcription factors in gene activation events during embryogenesis via the actions of specific cofactors.

[Key Words: LIM domain; coactivator; LIM and Otx homeodomain factors; protein-protein interaction; synergistic transcriptional activation]

Received March 4, 1997; revised version accepted April 22, 1997.

The LIM domain is a conserved cysteine- and histidine rich structure of two tandemly repeated zinc fingers, first identified in homeodomain transcription factors (for review, see Sánchez-García and Rabbitts 1994; Dawid et al. 1995). LIM domains were subsequently found in proteins that do not contain homeodomains, for example, in LIMonly proteins that contain essentially only one or several LIM domains, or in the LIM kinases LMK-1 and LMK-2 in which two LIM domains are associated with a protein kinase domain (Bernard et al. 1994; Mizuno et al. 1994). The LIM domain has been shown to be a protein-protein interaction domain (Feuerstein et al. 1994; Schmeichel and Beckerle 1994) and in LIM homeodomain factors, it has been implicated in the inhibition of DNA binding (Sánchez-García et al. 1993; Taira et al. 1994), but it has also been shown to be required for synergistic activation of genes by LIM homeodomain proteins acting with other transcription factors (German et al. 1992; Bach et al. 1995).

Members of the LIM homeodomain factor family exhibit distinct patterns of developmental expression. Isl-1 is expressed at high levels in hindbrain, forebrain, ven-

${ }^{1}$ Corresponding authors.

E-MAIL ibach@ucsd.edu;mrosenfeld@ucsd.edu; FAX (619) 534-8180. tral neural tube, dorsal root ganglia (DRG), and trigeminal ganglia. In the ventral neural tube, Isl-1 is the first marker of developing motor neurons (Ericson et al. 1992; Yamada et al. 1993) and a mutation of the Isl-1 gene leads to lack of motor neurons and interneurons (Pfaff et al. 1996). The LIM homeoprotein LH-2 is detected in discrete regions of the developing nervous system and in a subset of lymphoid tissues (Xu et al. 1993), whereas $\mathrm{P}-\mathrm{Lim} / \mathrm{Lhx} 3 / \mathrm{m}$-Lim3 is expressed highly and specifically early in development of the pituitary gland and the ventral neural tube (Seidah et al. 1994; Bach et al. 1995; Zhadanov et al. 1995). LH-2 and P-Lim/Lhx3 have been shown to be capable of binding to and activating the promoter of the glycoprotein hormone $\alpha$-subunit gene ( $\alpha \mathrm{GSU}$ ), a marker of early pituitary development, in transient transfection assays (Roberson et al. 1994; Bach et al. 1995). In the developing pituitary gland, P-Lim/Lhx3 mRNA is first detected at mouse embryonic (E) day 8.59.0 in the invagination of the somatic ectoderm in the roof of the oral epithelium, known as Rathke's pouch. The importance of P-Lim/Lhx3 in pituitary development was established by demonstrating that four of the five hormone-producing cell lineages in the anterior pituitary gland and expression of several markers including $\alpha$ GSU are lost in $L h x 3 / P$-Lim $(-/-)$ mice (Sheng et al. 1996). Several other homeodomain factors are highly expressed 
in early Rathke's pouch, including P-OTX/Ptxl, a homeoprotein belonging to the Otx class of transcription factors (Lamoneirie et al. 1996; Szeto et al. 1996) that is also able to activate $\alpha$ GSU gene expression. Because the expression patterns of P-Lim/Lhx3 and P-OTX/Ptxl overlap spatially and temporally in Rathke's pouch and thereafter in the developing pituitary gland, it is plausible that these two transcription factors may have a functional relationship (Szeto et al. 1996).

In this work we have used protein interaction screening to identify two highly homologous proteins that interact with the LIM domain of both P-Lim/Lhx3 and Isl-1. The mRNAs encoding these factors can be detected as early as E8.5 during mouse development and are expressed in regions that specifically express LIM homeodomain proteins. Although they cannot activate transcription from the $\alpha$ GSU gene, they are required for a synergistic activation of the $\alpha$ GSU gene by P-Lim/ Lhx3 and P-OTX/Ptxl and are therefore referred to as CLIM-1 and CLIM-2 (cofactor of LIM homeodomain proteins). These results suggest that members of this protein family function, at least in part, by enhancing the transcriptional efficiency of distinct classes of transcription factors by serving as coactivators critical for synergy, thus allowing the LIM and the Otx homeoproteins to be integrated into a larger transcriptional network.

\section{Results}

\section{A family of LIM-interacting proteins}

To define the factors interacting with the LIM domain of P-Lim/Lhx3, we screened mouse adult pituitary and E14.5 head $\lambda$ gtll expression libraries with a radioactively labeled LIM domain of P-Lim. Sequence analysis revealed that all 12 clones that we obtained by this method belonged to a gene family, with at least two members: CLIM-1, isolated from the pituitary library, and CLIM-2, isolated from the embryonic mouse head library. In addition to the P-Lim LIM domain, all clones bound to a radiolabeled protein fragment containing the LIM domain of Isl-1. All clones isolated contained sequences overlapping with the insert of the smallest clone (Fig. 1A), which limits the region in CLIM proteins that is required for the interactions with LIM domains to 109 amino acids. Full-length cDNAs of both transcripts, and one alternatively spliced form of CLIM-1 (CLIM-lb), were obtained by screening an embryonic pituitary library with the inserts of the $\lambda$ clones originally obtained. Except for a short stretch in their amino termini, CLIM$1 \mathrm{a}$ and CLIM-2 are highly homologous throughout their protein sequence, with a $75 \%$ overall amino acid identity (Fig. 1B), indicating that CLIM-1 and CLIM-2 define a family of LIM-interacting proteins. Comparison of
A

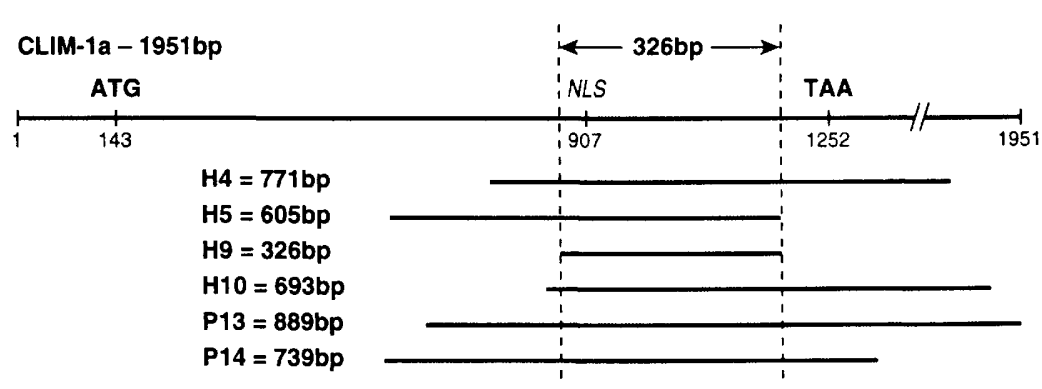

B
CLIM-1
CLIM-2
MS-STPHD-PFYSSPFG-PFYRRHTPYMVOPEYRIYZNARRI WLDRDVGPTIPMYP PTY LEPG IGRHTPYGNQY DYRIF EIMRLL
CLIM-1 DSRTEDSDNLWWDAFATEFFEDDATLTL SFCLEDGPKRYTIGRTLIPRYFSTVFEG CLIM-2 ONWIEECDNLWWDAFTIPEFFEDDAMLTITFCLEDGPKRYTIGRTLIPRY FRS IFEG

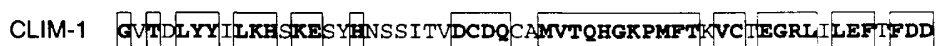
CLIM-2 CA

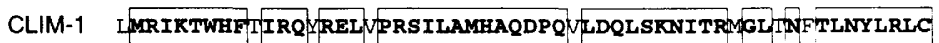
CLIM-2 MLRIKTWHFS IRQHRELIPRSILAMHAQDPQMLDQLSKNITRCGLSANSTLNYLRLC
CLIM-1 VILEPMQELMSRHKTYNLSPRDCLKTCLFQKWQRMVAPPAEFTR-QPTT
CLIM-2 VILEPMQELMSRHKTYSLSPRDCLKTCLFQKWQRMVAPPAEPARQQRS
CLIM-1b
CLIM-1a STSSTSNSSGGNITANSBG:
GLGAI PNCSINAGE RDGLCHS
CLIM-2 SGGSTMSSGGGNANM-sNS: PAASLSIATQVPDVMVVGEPTLMGGEFGDDER SPASTFAL S SQVPDVUVVGEPTLMGGEFGDEDER
CLIM-1b TAVTPSGQFKEKH *
CLIM-1a LITRLENTONDAANGMDDEEDFNNSPALGNNSPWNSKPPATQETKSENAPP-DASO* 373
CLIM-2 LITRLENTQFDAANGIDDEDSENNSPALGANSPWNSKPPSSQESTESEN-RPMQASQ*

Figure 1. Cloning of LIM domain-interacting proteins. $(A)$ Comparison of six representative $\lambda$ clones obtained in the protein interaction screening with a full-length CLIM-la cDNA, showing the size and location of the domain on CLIM-1 that is responsible for interaction with the LIM domain. A putative nuclear localization sequence (NLS) is indicated. (B) Amino acid sequence alignment of CLIM-1a, CLIM$1 \mathrm{~b}$, and CLIM-2. Identical residues are in bold letters and boxed. A hyphen indicates that this residue is not present. The putative nuclear localization domain is shaded, and an asterisk indicates a Stop codon. The amino-terminal 297 amino acids of CLIM-1a and CLIM-1b are identical. These sequences were deposited in the GenBank database under accession numbers U89487 (CLIM-1a), U89489 (CLIM-lb), and U89488 (CLIM-2). 
these two sequences with the sequence data base did not show significant homology to known proteins, and apart from a potential nuclear localization signal (NLS) (shaded in Fig. 1A), no known domains could be identified. However, subsequent to the initial characterization of these cDNAs, two other groups independently reported the sequence of a LIM domain-interacting protein NLI (Jurata et al. 1996) and Ldbl (Agulnick et al. 1996), both corresponding to the CLIM-2 sequence. Therefore, we refer to the proteins as CLIM-1 and CLIM-2/NLI/ Ldb1.

\section{CLIM-1 and CLIM-2/NLI/Ldb1 are transcribed in regions of specific LIM homeodomain expression}

The expression patterns of CLIM-1 and CLIM-2/NLI/ Ldb1 mRNAs were characterized by RNase protection assays, revealing that transcripts encoding both proteins were expressed in the pituitary gland, brain, testis, kidney, lung, skin, heart, liver, and trigeminal nerve (Fig. 2). The expression levels were not uniform, for example, CLIM-1 mRNA was expressed at highest levels in brain, trigeminal ganglia, and lung (Fig. 2A), whereas CLIM-2/ NLI/Ldb1 mRNA showed highest expression levels in the pituitary gland and skin (Fig. 2B). The abundance and the size of the two protected bands detected with the CLIM-1 probe indicated that the alternative spliced transcript encoded by CLIM-lb was expressed at even higher levels than the mRNA of the clone originally obtained, CLIM-1a, and that the ratio of both transcripts was tis-

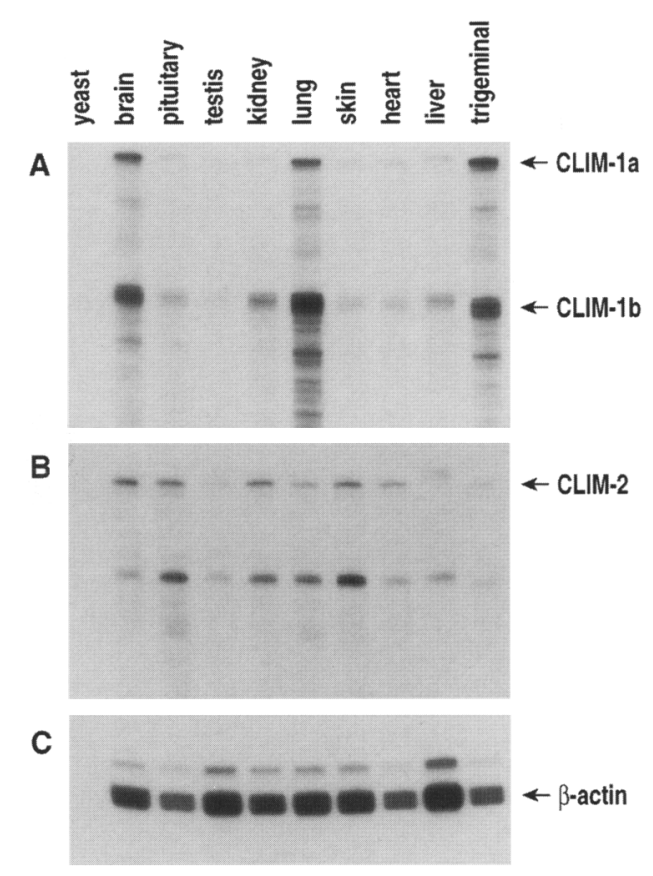

Figure 2. RNase protection experiments using RNA from adult mouse tissues. (A) RNase protection using a CLIM-1a-specific probe; $(B)$ RNase protection using a CLIM-2-specific probe; $(C)$ RNase protection using $\beta$-actin as probe. The expected bands in each experiment are indicated by arrows. sue dependent. A second protected band detected with the CLIM-2/NLI/Ldb1 probe suggests that an alternative transcript may also be synthesized for this gene (Fig. $2 \mathrm{~A}, \mathrm{BI}$.

To compare the early expression pattern of CLIM gene family members with P-Lim/Lhx3/m-Lim3 (Seidah et al. 1994; Bach et al. 1995; Zhadanov et al. 1995), Isl-1 (Karlsson et al. 1990), and LH-2 (Xu et al. 1993), we performed whole-mount in situ hybridizations on mouse embryos at stages E8.5, E9.0-9.5, and E11.5. The mRNAs encoding CLIM-1 and CLIM-2/NLI/Ldbl display overlapping expression patterns with LIM homeoproteins in mouse embryos (Fig. 3). At day E8.5, LIM homeoprotein transcripts were barely detectable, whereas CLIM-1 and CLIM-2/NLI/Ldbl mRNAs could be readily detected (data not shown). The hybridization patterns of CLIM-1 and CLIM-2 strongly resemble those of the LIM homeodomain genes at E9.0-9.5, with the highest mRNA expression detected in the telencephalon, the mesencephalon, and the metencephalon and with lower levels detected in the neural tube, the limbs, and in the DRG (Fig. 3G,I). At E9.0-9.5 P-Lim was detected in the rostral part of the telencephalon, in the roof of the mesencephalon, in the metencephalon, and in the olfactory epithelium (Fig. 3A); LH-2 transcripts were detected throughout the telencephalon, in the roof of the mesencephalon, in the metencephalon, in the olfactory epithelium, and the fore- and hindlimbs (Fig. 3C). In addition to a hybridization pattern similar to that of P-Lim and LH-2 in the head, specific Isl-1 expression was seen in the DRG and in the neural tube at stage E9.0-9.5 (Fig. 3E). In E11.5 embryos, the overlap in expression of CLIM mRNA with that of LIM homeobox genes was most striking in the developing midbrain and in the hindbrain where P-Lim, LH-2, and Isl-1 are expressed, in the forebrain where LH-2 and Isl-1 are expressed, and in the DRG where Isl-1 is expressed (Fig. 3B,D,F,H,J).

To further analyze the expression patterns of CLIM-1 and CLIM-2/NLI/Ldb1, we carried out in situ hybridizations on mouse embryonic head sections at stage E11.5 to postnatal day (P) 0 , using ${ }^{35} \mathrm{~S}$-labeled cRNA probes specific for CLIM-1 and CLIM-2/NLI/Ldb1. Both mRNAs are widely expressed at stage E17 and display a partially overlapping expression pattern in the mouse head (Fig. 4Al,B1). Both transcripts are highest in the developing neocortex, in the hippocampus, in the midbrain, in the medulla (Fig. $4 \mathrm{~A} 1, \mathrm{~B} 1$ and $4 \mathrm{~A} 2, \mathrm{~B} 2$ ), in the trigeminal ganglia (Fig. $4 \mathrm{Al}, \mathrm{Bl}$, and $\mathrm{A} 3, \mathrm{~B} 3\}$, in the nasal epithelium (Fig. 4A1,B1 and $A 4, B 4)$, in the submandibular gland (Fig. 4A1,B1, and $A 5, B 5$ ), in the ventral part of the neural tube (Fig. 4A6,B6), and in the DRG (Fig. $4 A 7, B 71$. The CLIM-1 probe hybridized in a more restricted fashion than the CLIM-2/NLI/Ldb1 probe, for example, in the neocortex CLIM-1 expression seems restricted to the cortical layers, whereas CLIM-2/NLI/ Ldb 1 mRNA is also detected closer to the intermediate and ventricular zones. In the olfactory bulb and certain striatal and thalamic brain regions, CLIM-2/NLI/Ldbl transcripts seemed to be more predominant.

From the expression studies, we conclude that low lev- 


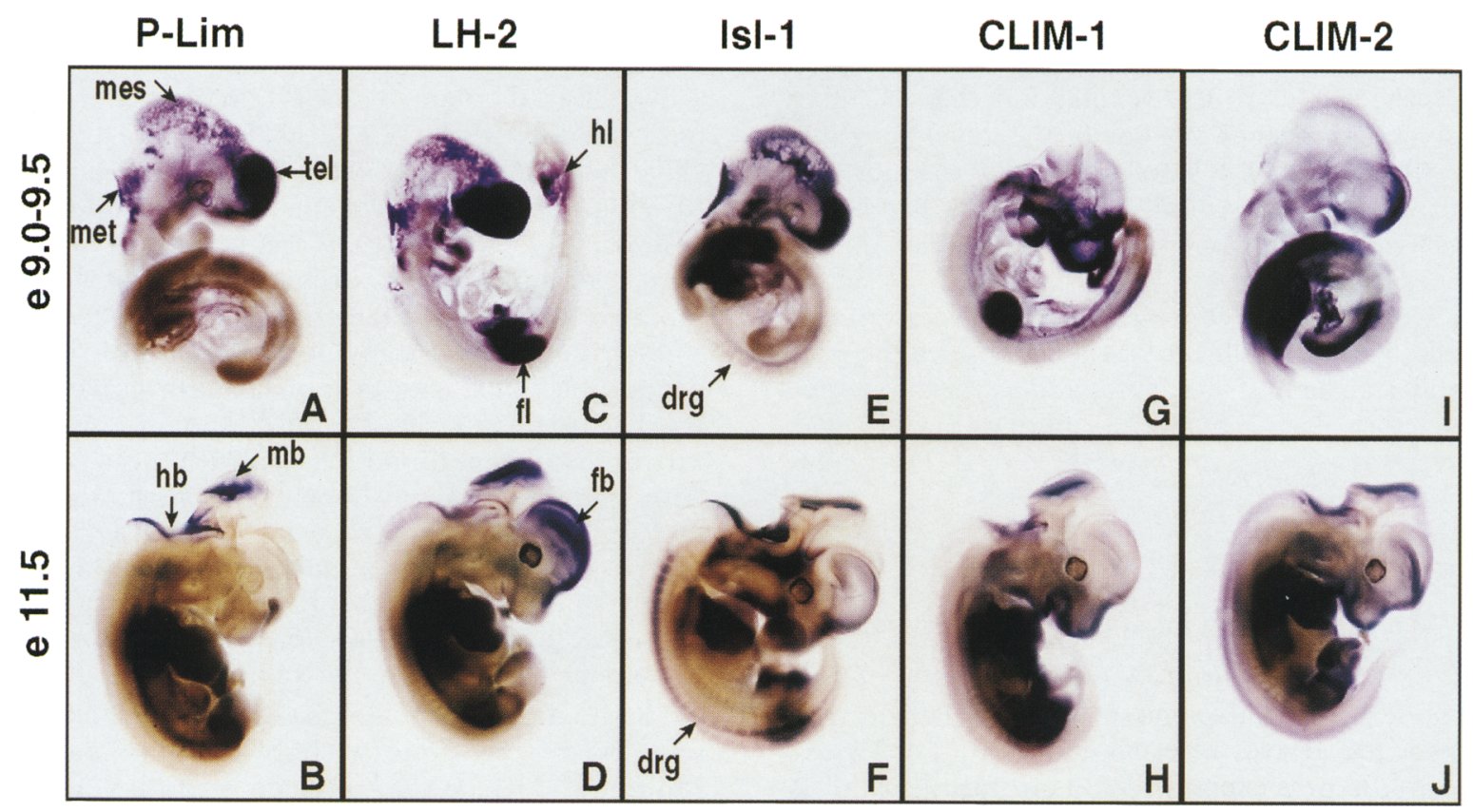

Figure 3. Comparison of the expression patterns of LIM homeoproteins with CLIM-1 and CLIM-2 by whole-mount in situ hybridizations on mouse embryos. $A, C, E, G$, and $I$ represent mouse embryos at stage E9.0-9.5, and $B, D, F, H$, and $/$ represent mouse embryos at stage E11.5. Embryos in $A$ and $B$ are hybridized with an P-Lim-specific probe, in $C$ and $D$ with a LH-2-specific probe, in $E$ and $F$ with a probe specific for Isl-1, in $G$ and $H$ with CLIM-1-specific probe, and in $I$ and $/$ with a CLIM-2-specific probe. The telencephalon (tel), mesencephalon (mes), metencephalon (met), forelimbs (fll), hindlimbs (hl) and dorsal root ganglia (drg) are indicated at stage E9.0-9.5, and at stage E11.5 the forebrain $(\mathrm{fb})$, midbrain $(\mathrm{mb})$, hindbrain $(\mathrm{hb})$ and dorsal root ganglia (drg) are indicated.
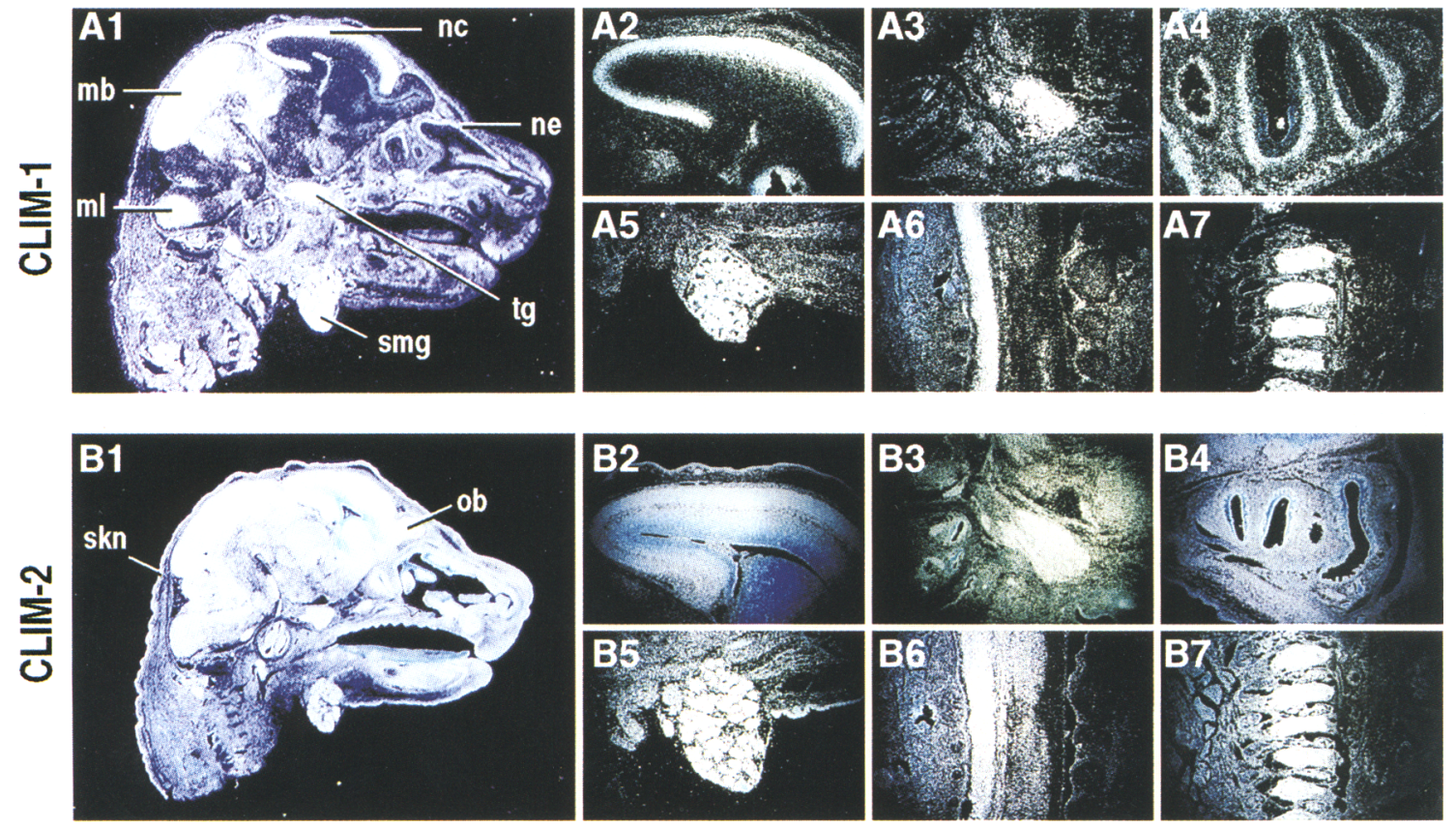

Figure 4. In situ hybridization experiments on head sections at mouse embryonic stage 17. (A) Hybridizations with a CLIM-1-specific probe; $(B)$ hybridizations with a CLIM-2-specific probe. $A 1$ and $B 1$ show the whole mouse head [ $|\mathrm{nc}|$ neocortex; $(\mathrm{mb}) \mathrm{midbrain} ;(\mathrm{ml})$ medulla; (smg) submandibular gland; (tg) trigeminal ganglia; (nel nasal epithelium; (ob) olfactory bulb; (skn) skin; enlargement of neocortex and hippocampus regions are shown in $A 2$ and $B 2$, the trigeminal ganglia in $A 3$ and $B 3$, the nasal epithelium $(A 4 / B 4)$, the submandibular gland $(A 5 / B 5)$, the neural tube $(A 6 / B 6)$, and DRG $\mid A 7 / B 7\}$. 
els of CLIM are initially detected broadly in E8.5 embryos before the detection of P-Lim/Lhx3, LH-2, and Isl-1 mRNAs. CLIM mRNA expression then becomes progressively stronger in regions of the developing embryo in which the LIM homeodomain transcripts are abundant. In addition, specific CLIM mRNA expression can be detected in regions of the embryo that do not overlap with expression of LIM homeodomain factors but which overlap with expression of other transcription factors like P-OTX/Ptxl. Interestingly, besides the pituitary gland, transient $\alpha$ GSU expression has been reported in the olfactory placode of mouse embryos (Kendall et al. 1994), overlapping with the expression of CLIM-2/NLI/ Ldb1.

The CLIM protein family interacts specifically with $P$-Lim/Lhx3 and P-OTX/Ptx 1

Having shown that the expression of CLIM transcripts overlapped that of LIM homeoproteins and P-OTX, we tested the protein-protein interaction capabilities of PLim, CLIM-1, and P-OTX in vitro. Immunoprecipitations were performed, using recombinant expressed proteins and specific antibodies directed against P-Lim and CLIM-1a. ${ }^{35}$ S-Labeled P-OTX can be immunoprecipitated with the CLIM-la antibodies upon addition of bacterially expressed, unlabeled CLIM-1a (Fig. 5A). Similarly, ${ }^{35}$ S-labeled P-Lim can be immunoprecipitated by CLIM- $1 \mathrm{a}$ antiserum in the presence of unlabeled CLIM1a. Both ${ }^{35}$ S-labeled P-OTX and CLIM-1a were immunoprecipitable using specific antiserum directed against $\mathrm{P}$ Lim, in the presence of unlabeled P-Lim. These experiments reveal that CLIM-la can interact with both P-Lim/Lhx3 and P-OTX and that P-Lim can interact with P-OTX.

We then tested whether P-Lim and P-OTX could form stable complexes with CLIM-1 proteins while bound to DNA by performing EMSA experiments with a ${ }^{32} \mathrm{P}$-radiolabeled oligonucleotide that contains the P-Lim and P-OTX binding sites of the $\alpha$ GSU promoter (Fig. $5 \mathrm{~B}, \mathrm{C}$ ). Full-length P-Lim, but not P-ALIM, formed an additional slower migrating complex when CLIM-la was added to the binding reaction (Fig. 5A). As expected, adding P-Lim antiserum inhibited formation of both complexes, whereas the addition of CLIM-1 antiserum inhibited specifically the slower migrating complex indicating that this complex contains both CLIM-1 and P-Lim. In a similar experiment CLIM-1b also proved capable to interact with P-Lim on DNA (data not shown). We then showed that bacterially expressed P-OTX is also able to associate with CLIM-la and CLIM-1b in EMSA experiments, demonstrating that this transcription factor is able to complex with CLIM factors on DNA, independently of the presence of LIM homeoproteins (Fig. 5C). When P-Lim, P-OTX, and CLIM-1a were mixed in such an experiment, several even slower migrating complexes appeared, suggesting the formation of complexes consisting of more than two proteins bound to DNA (data not shown). These results demonstrate that protein complexes con- sisting of at least P-Lim/CLIM-1 and P-OTX/CLIM-1 can form on DNA.

To define the domains of P-Lim that are responsible for interaction with CLIM-1, CLIM-2/NLI/Ldb1, and POTX, we tested the binding of ${ }^{35} \mathrm{~S}$-labeled $\mathrm{P}$-Lim proteins to glutathionine $S$-transferase (GST)-CLIM-1, GSTCLIM-2, and GST-P-OTX proteins (Fig. 6A). P-Lim proteins containing one or both zinc fingers of the LIM domain are capable of interacting with both CLIM and POTX. In contrast, a P-Lim protein lacking the entire LIM domain (P-ALIM) fails to interact with either CLIM or P-OTX, indicating that the LIM domain is both necessary and sufficient for interaction with these two factors. Although there may be differential contributions of LIM domain 1 and LIM domain 2 for CLIM-1, CLIM-2/NLI/ Ldb1, and P-OTX interactions, both are capable of sustaining interactions. The binding of the Isl-1 LIM domain to CLIM-1 was confirmed in this assay /data not shown).

The smallest clone obtained by the initial protein-protein screen corresponds to only 109 amino acids, thus limiting the region that interacts with the LIM domain to amino acids 233-342 of CLIM-1 (indicated in Fig. 6B) and to amino acids 236-344 of CLIM-2/NLI/Ldb1. To further map the protein interaction domain, we tested ${ }^{35}$ S-labeled mutant CLIM-1 proteins for their ability to bind to GST P-Lim and GST P-OTX (Fig. 6B). Whereas the CLIM-1a, CLIM-1b and a partial CLIM-1 (amino acids 1-296) protein bind well to P-Lim and P-OTX, the amino acids 1-226 fragment was not able to interact with both proteins (Fig. 6B). These results indicate that the carboxyl terminus of CLIM-1 mediates interactions with both proteins and defines a region of 63 amino acids (amino acids 233-296) as being crucial for efficient interaction with P-Lim and P-OTX.

Similarly, the domain of P-OTX responsible for interactions with P-Lim and CLIM-1 was mapped by analyzing ${ }^{35} \mathrm{~S}$-labeled P-OTX mutant proteins for their ability to bind to GST P-Lim and GST CLIM-la (Fig. 6C). The sequence from amino acids 150-281 in the carboxyl terminus of P-OTX was required for efficient interaction with P-Lim and CLIM-la.

\section{The CLIM proteins enhance the transactivation potential of P-Lim/Lhx3 and are required for $a$ transcriptional synergy between P-Lim/Lhx3 and P-OTX/PtX1}

To determine the cellular location of the CLIM-1a protein, we transfected a cytomegalovirus (CMV) promoterdriven CLIM-1 a construct into HeLa cells that was subsequently analyzed by immunostaining with a specific CLIM-la antiserum. CLIM-la was clearly localized to the nucleus, consistent with the presence of a putative NLS in its coding region (data not shown).

To begin to evaluate potential effects of the CLIM proteins on the transactivation functions of P-Lim, we cotransfected the -440 mouse $\alpha G S U$ promoter linked to the luciferase gene with plasmids expressing various combinations of P-Lim, P-OTX, and CLIM proteins. It 
A
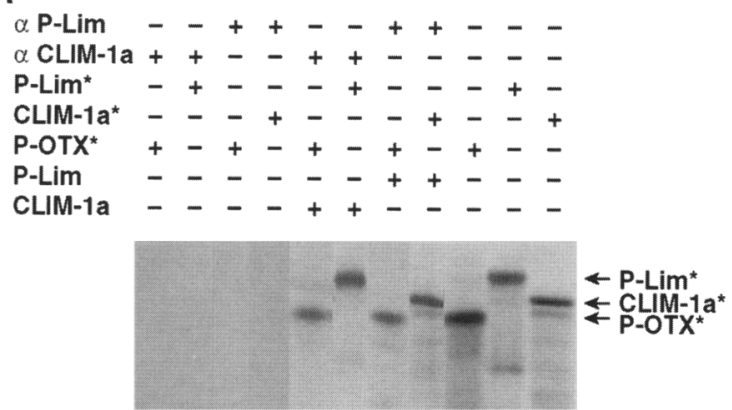

C

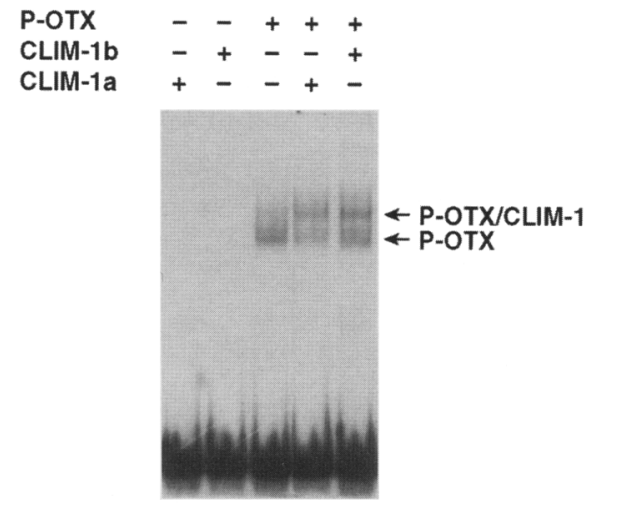

B

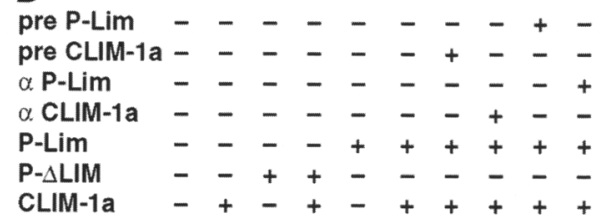

CLIM-1a

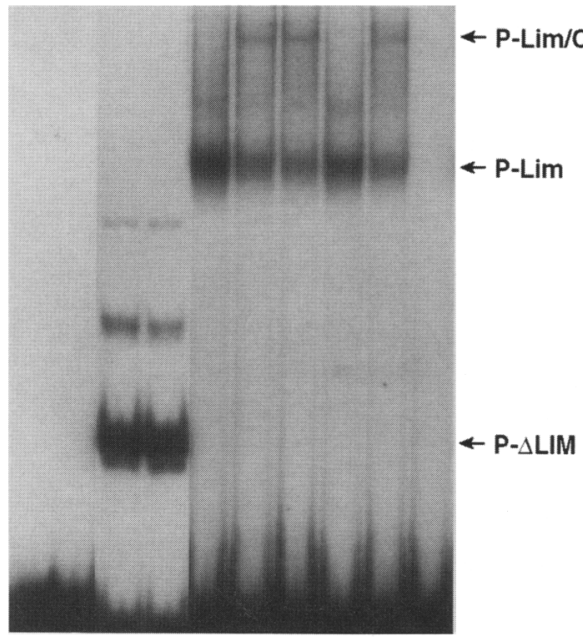

Figure 5. Interaction of CLIM-la with P-Lim and P-OTX. $|A|$ CLIM-1a interacts with P-Lim and P-OTX in immunoprecipitation assays. The proteins and the antisera $(\alpha)$ used are indicated. Asterisks indicate in vitro-translated, ${ }^{35} \mathrm{~S}$-labeled proteins. The input ${ }^{35} \mathrm{~S}$-labeled proteins represent $50 \%$ of the total amount added for the immunoprecipitations. (B) CLIM-la binds to P-Lim on DNA. Bacterially expressed CLIM-1a, P-ALIM, and P-Lim were used in EMSA experiments, and a double-stranded oligonucleotide containing the P-Lim and P-OTX binding sites was used as probe. For the P-JLIM protein, a 1:50 dilution was added to the binding reaction. The protein/DNA complexes are indicated by arrows. (C) CLIM-1 binds to P-OTX on DNA. The same oligonucleotide as in $B$ was used in EMSA experiments with bacterially expressed CLIM- $\mathrm{l}$ a, CLIM-1b, and P-OTX. In $A, B$, and $C$, all bands originate from the same gels, but some lanes were rearranged for a simpler presentation.

has been reported that $480 \mathrm{bp}$ of the $5^{\prime}$-flanking sequence of the mouse $\alpha \mathrm{GSU}$ promoter is sufficient to target expression into Rathke's pouch (Kendall et al. 1994). This promoter region contains at least one LIM homeodomain protein-binding site $(-342$ to -329$)$ that is recognized by P-Lim and LH-2 (Roberson et al. 1994; Bach et al. 1995) and one Otx-binding site $(-390$ to -383$)$ which is bound by P-OTX (Szeto et al. 1996). Full-length P-Lim activates this promoter 8 -fold, and a P-Lim mutant in which the entire LIM domain had been deleted (P-ALIM) activates 25-fold (Fig. 7A). Expression of CLIM-la alone had no effect on the $\alpha$ GSU promoter, but when cotransfected with full-length P-Lim, the transcriptional activation attained was similar to that observed with P-ALIM. In contrast, cotransfection of CLIM-la with P-ALIM showed no additional effect, indicating that the LIM domain is required for the coactivator function of CLIM-1a with PLim.

Because we have shown previously that P-Lim and POTX can independently activate the $\alpha$ GSU gene (Bach et al. 1995; Szeto et al. 1996), and because CLIM proteins can interact with both P-Lim and P-OTX on DNA, we tested the effect of CLIM proteins on P-OTX (Fig. 7A). P-OTX alone increased the trancription rates of the $\alpha$ GSU promoter by sevenfold, and cotransfection of CLIM-1 resulted in an additional threefold increase in transactivation. No additive activation effect was observed in cotransfections of full-length P-Lim with POTX. However, when P-Lim, P-OTX, and CLIM-la were cotransfected together, a strongly synergistic activation (134-fold) was measured. Surprisingly, this synergistic effect was also observed when P-ALIM was used instead of full-length P-Lim, demonstrating that in this case, the LIM domain was not required. Experiments using either CLIM-1b or CLIM-2/NLI/Ldb1 instead of CLIM-la gave similar results (data not shown), indicating that the synergistic effect is a general feature of the CLIM factors. Specificity of this effect was documented by cotransfections of CLIM-la or CLIM-1b with the POU domain transcription factors Pit-1 or Brn-4 with no potentiation observed in the activation of the thyroid-stimulating hormone $\beta$-subunit ( $\beta$-TSH), prolactin, or Pit-1 gene promoter (data not shown). Furthermore, CLIM-la or CLIM$1 \mathrm{~b}$ had no effect on the previously described synergy between P-Lim and Pit-1 (data not shown), indicating that the effect of CLIM-1a is both factor- and gene-specific. Together, these results show that CLIM proteins can function as coactivators for two distinct classes of ho- 
A

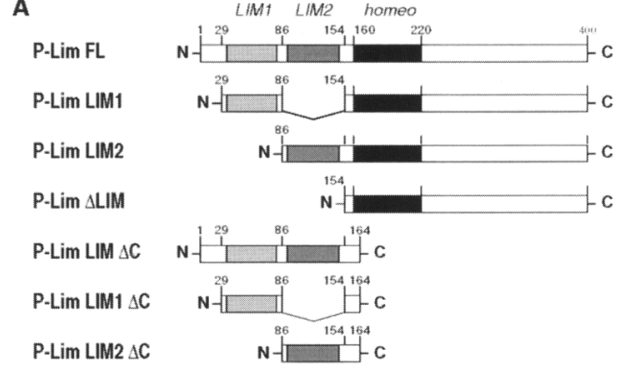

B

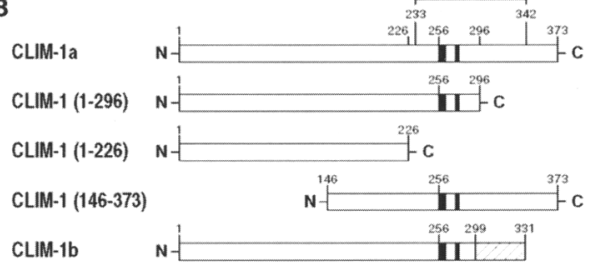

c

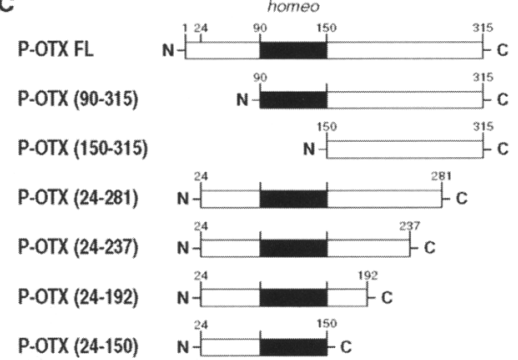

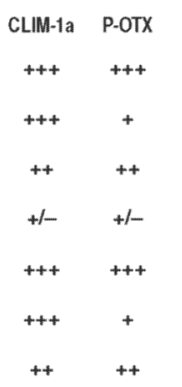
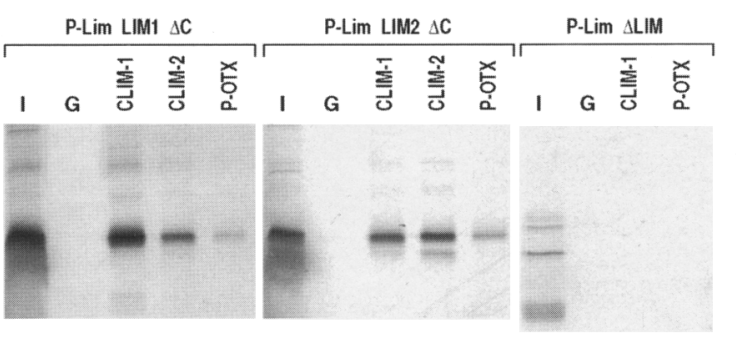

$+++$
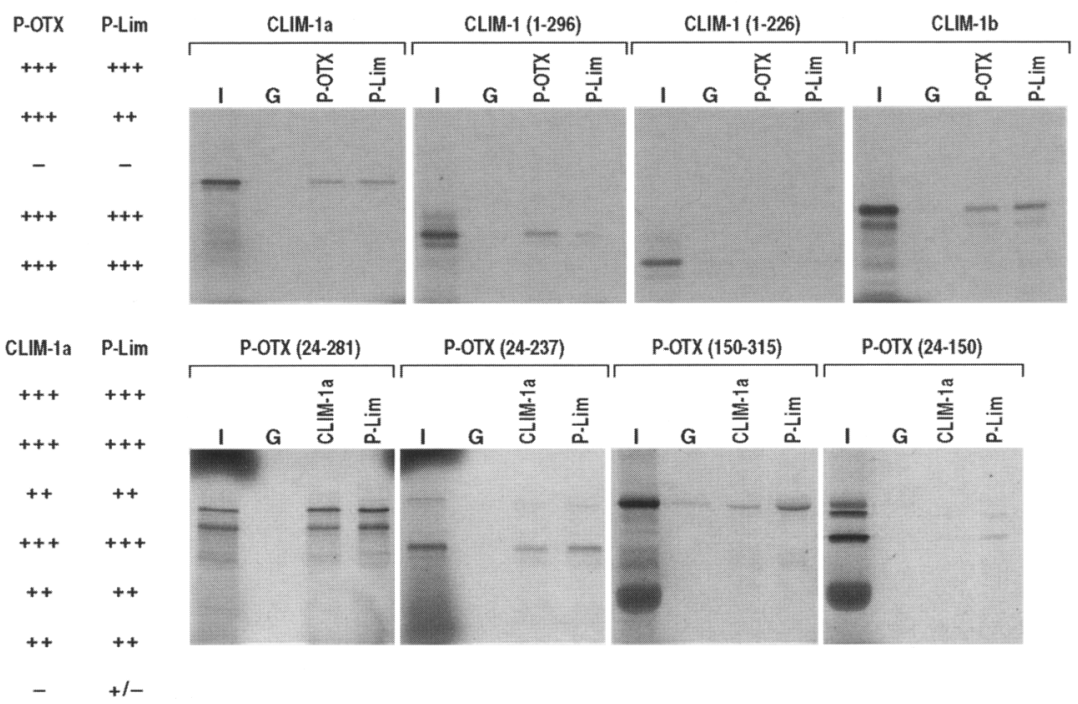

Figure 6. Mapping of the protein interaction domains in vitro. Full-length and deletion mutant ${ }^{35}$ S-labeled in vitro-translated proteins (left) were tested for their ability to bind to bacterially expressed GST fusion proteins. The relative strengths of interaction were scored from - (background level) to +++ (strong binding). (Right) Autoradiographs of representative experiments. The ${ }^{35} \mathrm{~S}$-labeled protein is indicated above the brackets and the GST fusion protein above each lane. Twenty percent of the total ${ }^{35} \mathrm{~S}$-labeled protein input (I) and binding to GST alone $(\mathrm{G})$ are shown. $(A)$ Identification of P-Lim domains that interact with GST-CLIM and GST-P-OTX fusion proteins. $(B)$ Identification of CLIM-1 domains that interact with GST-P-OTX and GST-P-Lim fusion proteins. $|C|$ Identification of P-OTX domains that interact with GST-CLIM-1a and GST-P-Lim fusion proteins.

meodomain transcription factors, represented by P-Lim/ Lhx3 and P-OTX/Ptx1, and that they can promote a striking synergy between these two factors.

\section{Discussion}

LIM homeodomain transcription factors exert essential functions in the determination of cell lineages during development. However, the specific role of the LIM domain in these factors has been unclear. Several features have been attributed to this structure. In addition to being inhibitory for binding of the holoprotein to DNA (e.g., Sanchez-Garcia et al. 1993), the LIM domain has been shown to be required for synergistic transcriptional activation conferred by LIM homeodomain proteins together with other transcription factors /German et al. 1992; Bach et al. 1995). Furthermore, it has been established that the LIM domain is a structure mediating protein-protein interactions (e.g., Feuerstein et al. 1994; Schmeichel and Beckerle 1994). The isolation of cDNAs encoding the two highly homologous proteins CLIM-1 and CLIM-2/NLI/Ldbl, based on their ability to interact with the LIM domain of P-Lim/Lhx3, has permitted a further clarification of the functions of the LIM domain. The observation that both proteins can interact with the LIM domains of P-Lim/Lhx3 and Isl-1 suggests that members of the CLIM protein family may interact with many or even most of the LIM homeodomain proteins. This hypothesis is supported by the temporal and spatial expression patterns of CLIM-1 and CLIM-2/NLI/Ldbl, which is overlapping with that of LIM homeodomain factors in the mesencephalon, metencephalon, and telencephalon during mouse development. Even at later developmental stages, the regions of highest CLIM-1 and CLIM-2/NLI/Ldbl gene expression correspond with regions of specific expression of different LIM homeoprotein members. Particularly striking are the parallel expression patterns of Isl-1 (Thor et al. 1991) and CLIM transcripts in the DRG and trigeminal ganglia, where, to our knowledge, Isl-1 is the only expressed LIM homeodomain protein.

LIM domain-dependent CLIM interaction increased 
A
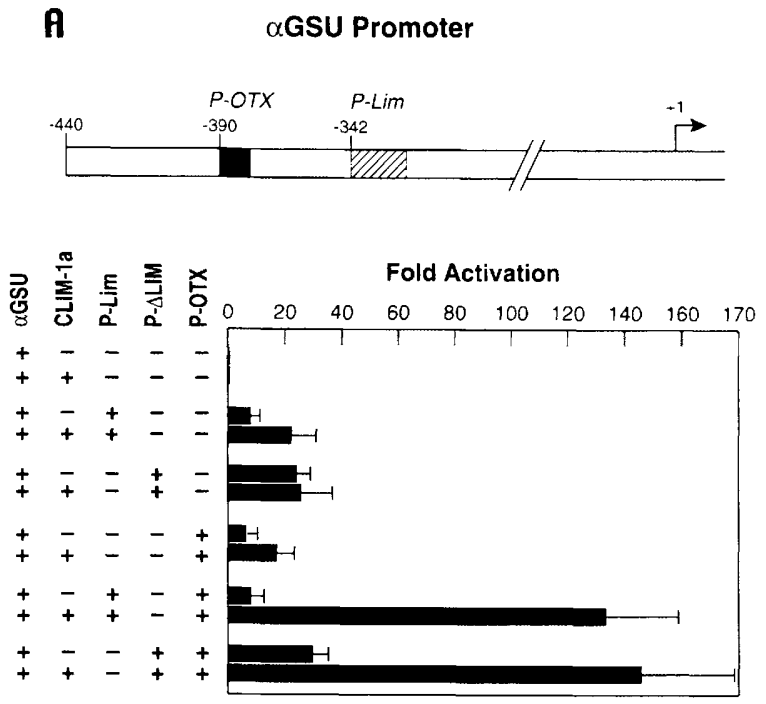

B

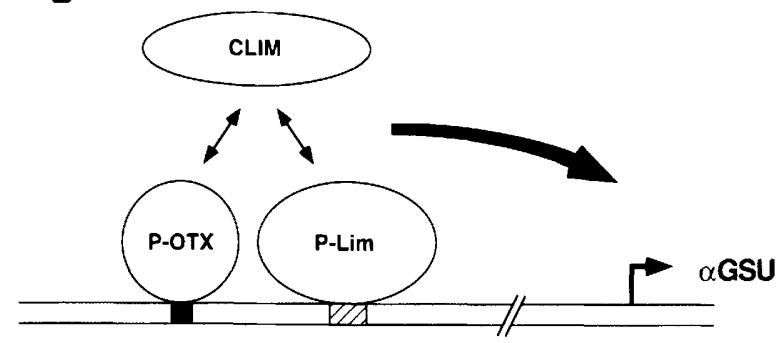

Figure 7. (A) Activation levels of the $\alpha \mathrm{GSU}$ promoter linked to the luciferase gene in cotransfections. A schematic of the promoter, including the P-Lim and P-OTX binding sites, is shown at the top. Results are expressed as fold activation, mean \pm standard deviation of the mean, compared to activity of the $\alpha \mathrm{GSU}$ promoter in the presence of an empty CMV expression plasmid. The combination of cotransfected CMV-driven expression plasmids and the $\alpha \mathrm{GSU}$ reporter plasmid are indicated. $\langle B\rangle$ Model of CLIM action on the $\alpha$ GSU promoter. For synergistic activation all three proteins are required.

the transactivation potential of P-Lim holoprotein by threefold, similar to the activity of LIM domain-deleted protein. These findings are analogous to a study of the Xenopus LIM homeodomain factor Xlim1, which is expressed in Spemann's organizer at the gastrula stage (Taira et al. 1992). Xlim l constructs in which both LIM domains are mutated or deleted, can induce secondary axis formation with associated muscle and neural differentiation, when injected into blastula-stage frog embryos, whereas Xlim 1 holoprotein has only a small effect (Taira et al. 1994). Xenopus Ldbl protein proved to enhance the activity of the Xlim 1 holoprotein in inducing a secondary axis upon coinjection into blastula-stage frog embryos (Agulnick et al. 1996), arguing for a negative role of the LIM domain.

We have found that CLIM-1 also interacts specifically with a member of the Otx family of transcription factors (P-OTX/Ptx1), consistent with a model where P-Lim, POTX, and CLIM-1 can form complexes on DNA. Mem- bers of the Otx class of the bicoid-related homeobox transcription factors have been proven to play essential roles in vertebrate development. For example, Otx-2 is required for anterior head structures (Acampora et al. 1995; Matsuo et al. 1995; Ang et al. 1996), Prop-1 mutations appear to be responsible for the Ames mouse dwarf phenotype (Sornson et al. 1996) and mutations in the RIEG gene, which encodes the solurshin protein, cause Rieger syndrome (Semina et al. 1996). In the homeodomain and its carboxy-terminal half, solurshin shares extensive sequence homology with P-OTX/Ptxl, which is highly expressed in Rathke's pouch and developing pituitary gland (Lamonerie et al. 1996; Szeto et al. 1996). The similar pattern of expression of P-Lim/Lhx3 and P-OTX/ Ptxl throughout the developing pituitary gland (Szeto et al. 1996), the ability of both proteins to activate the $\alpha$ GSU gene in transient transfection assays (Bach et al. 1995; Szeto et al. 19961, and the observations that CLIM mRNA is found in regions of P-OTX expression has led us to consider a functional relationship between the two factors. Whereas cotransfection of P-Lim with P-OTX showed no additive activation effect on the $\alpha$ GSU gene, they produced a strong synergistic activation in the presence of CLIM-1. The fact that the CLIM protein family can also increase the transactivation potential of P-OTX, taken together with the observation that P-OTX and CLIM mRNAs appear to have a similar, overlapping expression pattern in regions such as the submandibular gland, suggests the possibility that CLIM proteins may also modulate functions of members of the P-OTX protein family independently of LIM homeodomain proteins.

Confirming previous reports that the LIM domain is inhibitory for DNA binding of full-length LIM homeoprotein (e.g., Sánchez-García et al. 1993), the deletion of the LIM domain of P-Lim increased binding to its cognate DNA site. Our experiments revealed that CLIM-1a failed to increase binding of P-Lim holoprotein to DNA, as assayed by EMSA with various amounts of P-Lim and CLIM-1. Furthermore, DNase I footprinting experiments using different concentrations of bacterially expressed full-length P-Lim, P-JLIM, P-OTX, and CLIM-1 proteins on the $\alpha \mathrm{GSU}$ promoter region, which encompasses the binding sites for both proteins, did not reveal any change in the footprint patterns of P-Lim or P-OTX on their respective sites in the presence of CLIM-1 (data not shown). Collectively these findings suggest that release of the inhibitory effect of the LIM domain did not reflect effects on DNA binding by P-Lim.

The results of our EMSA experiments, DNase I footprints, and the observation that CLIM proteins are able to increase the transcriptional activity not only of P-Lim but also of P-OTX (Fig. 7A) argue against a mechanism in which the sole function of CLIM proteins is to increase DNA binding of the associated factor. Furthermore, the synergistic activation observed upon cotransfection of P-JLIM, P-OTX, and CLIM-1 cannot be explained by a model in which the only function of the CLIM proteins is to relieve the inhibition of DNA binding conferred by the LIM domain. To explain the function of the CLIM 
proteins we prefer a model in which synergy is dependent on the presence of all three proteins in a spatial vicinity, with the CLIM protein associated with P-Lim/ Lhx3 and/or P-OTX/Ptxl (Fig. 7B). Thus, our results suggest that the CLIM proteins are likely to exert at least a component of their biological effects by enhancing transcription and promoting synergism rather than by relieving the inhibition on DNA binding by the LIM domain.

Our in situ hybridization experiments show that both CLIM-1 and CLIM-2/NLI/Ldbl mRNAs are highly expressed in the ventral part of the developing neural tube (Fig. 4A5,B5). As LIM homeobox genes are expressed in a temporal and spatial gradient along the developing neural tube, subclasses of motor neurons can be distinguished by the expression of a distinct combination of LIM homeobox genes, and a gradient of LIM homeoprotein expression has been reported to be responsible for motor neuron fate specification (Tsuchida et al. 1994; Appel et al. 1995; Pfaff et al. 1996). It is tempting to speculate that the CLIM proteins will play important roles in LIM homeodomain protein-dependent motorneuron specification. The similar phenotype of mice (-/-) for mLim-1 (Shawlot and Behringer 1995) and Otx-2 (Acampora et al. 1995; Matsuo et al. 1995; Ang et al. 1996) has prompted these investigators to propose a functional relationship between the LIM and the Otx homeoprotein gene families. The fact that CLIM-encoding mRNAs show a widely overlapping expression pattern with Otxl and Otx 2 in the developing mouse brain (Simeone et al. 1992) suggests that the CLIM protein family may play critical roles in the functional relationships of LIM homeoproteins and additional Otx factors.

In summary, we propose that members of the CLIM protein family function as integrator molecules, leading to a synergistic activation of genes expressed in a tissuespecific manner.

\section{Materials and methods}

\section{Cloning of CLIM-1 and CLIM-2/NLI/Ldb1}

Protein-protein interactions screenings were carried out essentially as described by Kaelin et al. (1992). Briefly, an XbaI-NotI fragment containing both LIM domains of P-Lim was inserted into the GST bacterial expression vector GST-KGK. The resulting GST fusion protein was radioactively labeled with protein kinase $A$ and $\left[\gamma_{-}{ }^{32} \mathrm{P}\right] \mathrm{ATP}$ (NEN) and used to screen adult mouse pituitary and E14.5 mouse head $\lambda$ gt 11 expression libraries under denaturing/renaturing and nondenaturing conditions. The inserts of positive clones were subcloned into the Bluescript vector pBKS and analyzed by DNA sequencing. Full-length cDNAs were obtained by screening an embryonic pituitary cDNA library (Sornson et al. 1996) using the random primed ${ }^{32} \mathrm{P}$-radiolabeled inserts from the clones obtained in the initial proteinprotein screening as probes.

\section{RNase protection assays and in situ hybridization}

RNase protection assays and in situ hybridizations were performed using cDNA clones (P14 and $\mathrm{H} 5$ in Fig. 1A, corresponding to CLIM-1a and CLIM-2, respectively) as templates for T7and T3-directed cRNA synthesis, generating labeled antisense probes of 739 and 605 nucleotides, respectively. The RNase protection experiments were done as described previously (Andersen et al. 1993). In situ hybridization of 20- $\mu$ m sagittal sections of mouse embryos (E11.5, E15.5, E17, and P0\}, fixed in $10 \%$ buffered formaline, was performed as described previously (Simmons et al. 1990). Whole-mount in situ hybridization studies on mouse embryos E8.5, E9.0-9.5, and E11.5 were carried out essentially as described by Wilkinson (1993). The LIM homeodomain protein antisense probes used for the whole-mount in situ hybridizations were cDNA regions corresponding to amino acids 221-400 of P-Lim (Bach et al. 1995), amino acids 23-426 of LH-2 (Xu et al. 1993), and amino acids 118-348 of Isl-1 (Karlson et al. 1990).

\section{Antibody generation, immunoprecipitations, and} DNA-binding assays

Polyclonal antibodies were raised against the carboxyl termini of the P-Lim protein (amino acids 221-400) and CLIM-1a (amino acids 146-373). Rabbits and guinea pigs were immunized with the respective GST fusion proteins, which were expressed in Escherichia coli and partially purified on 10\% SDS polyacrylamide gels. Immunoprecipitations were carried out as described previously (Harlow and Lane 1988). The radiolabeled proteins were obtained by in vitro transcription/translation with Promega rabbit reticulocyte lysates and $\left[{ }^{35} \mathrm{~S}\right]$ methionine and mixed with bacterial expressed proteins and specific antiserum.

Protein-mediated gel shift assays, were carried out as described previously (Ingraham et al. 1988) with the modifications that the binding reaction was carried out on ice and that the gels were run at $4^{\circ} \mathrm{C}$. Two microliters of a 1:10 serum dilution was added to the binding reaction and incubated on ice for $10 \mathrm{~min}$. The sequence of the oligonucleotide used in this experiment was 5'-GAAATAATGTAATCCTGAAAATATATCAGGTACTTAGCTAATTAAATGTGCTACT-3' (sense strand) encompassing the P-Lim and the P-OTX recognition sites on the $\alpha$ GSU promoter.

\section{In vitro protein-protein interaction and transfection assays}

For the in vitro protein-protein interaction assays, cDNAs encoding the P-Lim mutants P-Lim LIM $\Delta \mathrm{C}$, P-Lim LIM1 $\Delta \mathrm{C}$, and P-Lim LIM2 $\Delta C$ were synthesized using PCR. The amplified fragments were inserted into the pCITE vector (Novagen). The

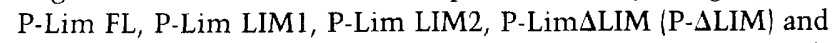
the GST-P-Lim fusion constructs are described in Bach et al. (1995). PCR products of full-length CLIM-1a and CLIM-1b were ligated in-frame into the $X$ hol-XbaI sites of the pGEX-KG to yield a GST fusion protein. $\left[{ }^{35} S\right]$ methionine-labeled in vitrotranslated protein was used for the in vitro protein-protein interaction assays as described previously (Bach et al. 1995). CLIM-1 carboxy-terminal deletions were obtained by AvaI (amino acids 1-226) and KpnI (amino acids 1-296) digestion and subsequent in vitro transcription/translations. The amino-terminal deletion mutant (amino acids 146-373) was cloned as an EcoRI fragment in-frame into the pCITE vector. The P-OTX carboxy-terminal deletion mutants were obtained by XhoI (amino acids 24-271), SphI (amino acids 24-237), and NarI (amino acids 24-192) digestions and subsequent in vitro transcription/translations. The P-OTX mutants /amino acids 90$315,150-315$, and 24-150) and the GST-P-OTX fusion construct were described previously (Szeto et al. 1996). All in vitro transcription/translations were carried out using the Promega in vitro transcription/translation kit and $\left[{ }^{35} S\right]$ methionine.

In vitro culture and transient transfections of HeLa cells were carried out as described previously (Rhodes et al. 1993). Typi- 
cally, $2 \mu \mathrm{g}$ of reporter plasmid and $1 \mu \mathrm{g}$ of effector plasmid were added as calcium phosphate precipitates per $60-\mathrm{mm}$ plate. The transfected plasmids were $\alpha \mathrm{GSU}$-luciferase reporter plasmid, CMV promoter-driven P-Lim, P-Lim SLIM (Bach et al. 1995), P-OTX (Szeto et al. 1996), and CLIM-1a, CLIM-1b, and CLIM$2 / \mathrm{NLI} / \mathrm{Ldb} 1$. For the full-length CLIM-1a, CLIM-b, and CLIM2/NLI/Ldbl expression plasmids HindIII-Xbal-digested fragments were cloned into pcDNA3 (Invitrogen).

\section{Acknowledgments}

We are grateful to $\mathrm{C}$. Nelson for expert cell culture, to $\mathrm{S}$. O'Connell, D. Szeto, and Drs. R. McEvilly, A. Ryan, B. Gloss, C. Kioussi, and T. Heinzel for providing critical reagents and advice. H.P.O. was supported by a Lucille Markey predoctoral fellowship, and B.A. is supported by an American Cancer Society Institutional grant and a Clinical Investigator Award (DK02411) from the National Institutes of Health (NIH). M.G.R. is an Investigator with the Howard Hughes Medical Institute. This work was supported by a grant from the NIH.

The publication costs of this article were defrayed in part by payment of page charges. This article must therefore be hereby marked "advertisement" in accordance with 18 USC section 1734 solely to indicate this fact.

\section{References}

Acampora, D., S. Mazan, Y. Lallemand, V. Avantaggiato, M. Maury, A. Simeone, and P. Brulet. 1995. Forebrain and midbrain regions are deleted in Otx2-/- mutants due to a defective anterior neuroectoderm specification during gastrulation. Development 121: 3279-3290.

Agulnick, A.D., M. Taira, J.J. Breen, T. Tanaka, I.B. Dawid, and H. Westphal. 1996. Interactions of the LIM-domain-binding factor Ldb1 with LIM homeodomain proteins. Nature 384: $270-272$.

Andersen, B., M.D. Schonemann, R.V. Pearse II, K. Jenne, J. Sugarman, and M.G. Rosenfeld. 1993. Brn-5 is a divergent POU domain factor highly expressed in layer IV of the neocortex. I. Biol. Chem. 268: 23390-23398.

Ang, S.L., O. Jin, M. Rhinn, N. Daigle, L. Stevenson, and J. Rossant. 1996. A targeted mouse Otx2 mutation leads to severe defects in gastrulation and formation of axial mesoderm and to deletion of rostral brain. Development 122: $243-252$.

Appel, B., V. Korzh, E. Glasgow, S. Thor, T. Edlund, I.B. Dawid, and J.S. Eisen. 1995. Motoneuron fate specification revealed by patterned LIM homeobox gene expression in embryonic zebrafish. Development 121: 4117-4125

Bach, I., S.J. Rhodes, R.V. Pearse II, T. Heinzel, B. Gloss, K.M. Scully, P.E. Sawchenko, and M.G. Rosenfeld. 1995. P-Lim, a LIM homeodomain factor, is expressed during pituitary organ and cell commitment and synergizes with Pit-1. Proc. Natl. Acad. Sci. 92: 2720-2724.

Bernard, O., S. Ganiatsas, G. Kannourakis, and R. Dringen. 1994. Kiz-1, a protein with LIM zinc finger and kinase domains, is expressed mainly in neurons. Cell Growth Differ. 5: $1159-1171$.

Dawid, I.B., R. Toyama, and M. Taira. 1995. LIM domain proteins. C.R. Acad. Sci. Ser. III 318: 295-306.

Ericson, J., S. Thor, T. Edlund, T.M. Jessell, and T. Yamada. 1992. Early stages of motor neuron differentiation revealed by expression of homeobox gene Islet-1. Science 256: 155160.

Feuerstein, R., X. Wang, D. Song, N.E. Cooke, and S.A. Lieb- haber. 1994. The LIM/double zinc-finger motif functions as a protein dimerization domain. Proc. Natl. Acad. Sci. 91: 10655-10659.

German, M.S., J. Wang, R.B. Chadwick, and W.J. Rutter. 1992. Synergistic activation of the insulin gene by a LIM-homeo domain protein and a basic helix-loop-helix protein: Building a functional insulin minienhancer complex. Genes \& Dev. 6: 2165-2176.

Harlow, E. and D. Lane. 1988. Antibodies. A laboratory manual, 2nd ed. Cold Spring Harbor Laboratory, pp. 421446. Cold Spring Harbor, NY.

Ingraham, H.A., R.P. Chen, H.J. Mangalam, H.P. Elsholtz, S.E. Flynn, C.R. Lin, D.M. Simmons, L. Swanson, and M.G. Rosenfeld. 1988. A tissue-specific transcription factor containing a homeodomain specifies a pituitary phenotype. Cell 50: $519-529$.

Jurata, L.W., D.A. Kenny, and G.N. Gill. 1996. Nuclear LIM interactor, a rhombotin and LIM homeodomain interacting protein, is expressed early in neuronal development. Proc. Natl. Acad. Sci. 93: 11693-11698.

Kaelin, W.G., W. Krek, W.R. Sellers, J.A. DeCaprio, F. Ajchenbaum, C.S. Fuchs, T. Chittenden, Y. Li, P.J. Farnham, M.A. Blanar, D.M. Livingston, and E.K. Flemington. 1992. Expression cloning of a retinoblastoma-binding protein with E2Flike properties. Cell 70: 351-364.

Karlsson, O., S. Thor, T. Norberg, H. Ohlsson, and T. Edlund. 1990. Insulin gene enhancer binding protein Isl-1 is a member of a novel class of proteins containing both a homeo and a cys-his domain. Nature 344: 879-882.

Kendall, S.K., D.F. Gordon, T.S. Birkmeier, D. Petrev, V.D. Sarapura, S. O'Shea, W.M. Wood, R.V. Lloyd, E.C. Ridgway, and S.A. Camper. 1994. Enhancer-mediated high level expression of mouse pituitary glycoprotein hormone alpha-subunit transgene in thyrotropes, gonadotropes, and developing pituitary gland. Mol. Endocrinol. 8: 1420-1433.

Lamoneirie, T., J.J. Tremblay, C. Lanctot, M. Therrien, Y. Gauthier, and J. Drouin. 1996. Ptx-1, a bicoid-related homeobox transcription factor involved in transcription of the pro-opiomelanocortin gene. Genes \& Dev. 10: 1284-1295

Matsuo, I., S. Kuratani, C. Kimura, N. Takeda, and S. Aizawa. 1995. Mouse Otx2 functions in the formation and patterning of rostral head. Genes \& Dev. 9: 2646-2658.

Mizuno, K., I. Okano, K. Ohashi, K. Nunoue, K. Kuma, T. Miyata, and T. Nakamura. 1994. Identification of a human cDNA encoding a novel protein kinase with two repeats of the LIM/double zinc finger motif. Oncogene 9: 1605-1612.

Pfaff, S.L., M. Mendelsohn, C.L. Stewart, T. Edlund, and T.M. Jessell. 1996. Requirement for LIM homeobox gene Isll in motor neuron generation reveals a motor neuron-dependant step in interneuron differentiation. Cell 84: 309-320.

Rhodes, S.J., R. Chen, G.E. DiMattia, K.M. Scully, K.A. Kalla, S.C. Lin, V.C. Yu, and M.G. Rosenfeld. 1993. A tissue-specific enhancer confers Pit-1-dependent morphogen inducibility and autoregulation on the Pit-1 gene. Genes \& Dev. 7: 913-932.

Roberson, M.S., W.E. Schoderbek, G. Tremml, and R.A. Maurer. 1994. Activation of the glycoprotein hormone alpha-subunit promoter by a LIM-homeodomain transcription factor. Mol. Cell. Biol. 14: 2985-2993.

Sánchez-García, I. and T.H. Rabbitt. 1994. The LIM domain: A new structural motif found in zinc-finger proteins. Trends Genet. 10: 315-320.

Sánchez-García, I., H. Osada, A. Forster, and T.H. Rabbitts. 1993. The cysteine-rich LIM domains inhibit DNA binding by the associated homeodomain in Isl-1. EMBO J. 12: 42434250 . 
Bach et al.

Schmeichel, K.L. and M.C. Beckerle. 1994. The LIM domain is a modular protein-binding interface. Cell 79: 211-219.

Seidah, N.G., J.-C. Barale, M. Marcinkiewicz, M.-G. Mattei, R. Day, and M. Chretien. 1994. The mouse homeoprotein mLIM-3 is expressed in cells derived from the neuroepithelium and persists in adult pituitary. DNA Cell Biol. 13: $1163-1180$.

Semina, E.V., R. Reiter, L.J. Leysens, W.L.M. Alward, K.W. Small, N.A. Datson, J. Siegel-Bartelt, D. Bierke-Nelson, P. Bitoun, B.U. Zabel, J.C. Carey, and J.C. Murray. 1996. Cloning and characterization of a novel bicoid-related homeobox transcription factor gene, RIEG, involved in Rieger syndrome. Nature Genet. 14: 392-399.

Shawlot, W. and R.R. Behringer. 1995. Requirement for Lim 1 in head-organizer function. Nature 374: 425-430.

Sheng, H.Z., A.B. Zhadanov, B. Mosinger, T. Fujii, S. Bertuzzi, A. Grinberg, E.J. Lee, S.-P. Huang, K.A. Mahon, and H. Westphal. 1996. The LIM homeobox gene Lhx-3 is essential for the specification and proliferation of pituitary cell lineages. Science 256: 1555-1560.

Simeone, A., D. Acampora, M. Gulisano, A. Stornaiuolo, and E. Boncinelli. 1992. Nested expression domains of four homeobox genes in developing rostral brain. Nature 358: 687690.

Simmons, D.M., J.W. Voss, H.A. Ingraham, J.M. Holloway, R.S. Broide, M.G. Rosenfeld, and L.W. Swanson. 1990. Pituitary cell phenotypes involve cell-specific Pit- 1 mRNA translation and synergistic interactions with other classes of transcription factors. Genes \& Dev. 4: 695-711.

Sornson, M.W., W. Wu, J.S. Dasen, S.E. Flynn, D.J. Norman, S.M. O'Connell, I. Gukovsky, C. Carrière, A.K. Ryan, A.P. Miller, L. Zuo, A.S. Gleiberman, B. Andersen, W.G. Beamer, and M.G. Rosenfeld. 1996. Pituitary lineage determination by the Prophet of Pit-1 homeodomain factor defective in Ames dwarfism. Nature 384: 327-333.

Szeto, D.P., A.K. Ryan, S.M. O'Connell, and M.G. Rosenfeld. 1996. P-OTX: A Pit-1-interacting homeodomain factor expressed during anterior pituitary gland development. Proc. Natl. Acad. Sci. 93: 7706-7710.

Taira, M., M. Jamrich, P.J. Good, and I.B. Dawid. 1992. The LIM domain-containing homeobox gene Xlim-1 is expressed specifically in the organizer region of Xenopus gastrula embryos. Genes \& Dev. 6: 356-366.

Taira, M., H. Otani, J.-P. Saint-Jeannet, and I.B. Dawid. 1994. Role of the LIM class homeodomain protein Xlim-1 in neural and muscle induction by the Spemann organizer in Xenopus. Nature 372: 677-679.

Thor, S., J. Ericson, T. Brannstrom, and T. Edlund. 1991. The homeodomain LIM protein Isl-1 is expressed in subsets of neurons and endocrine cells in the adult rat. Neuron 7:881889.

Tsuchida, T., M. Ensini, S.B. Morton, M. Baldassare, T. Edlund, T.M. Jessell, and S.L. Pfaff. 1994. Topographic organization of embryonic motor neurons defined by expression of LIM homeobox genes. Cell 79: 957-970.

Wilkinson, D.G. 1993. In situ hybridization. In Essential developmental biology: A practical approach (ed. C.D. Stern and W.H. Holland), pp. 259-274. IRL, Oxford, UK.

$\mathrm{Xu}$, Y., M. Baldassare, P. Fisher, G. Rathbun, E.M. Oltz, G.D. Yancopoulos, T.M. Jessell, and F.W. Alt. 1993. LH-2: A LIM/ homeodomain gene expressed in developing lymphocytes and neural cells. Proc. Nat1. Acad. Sci. 90:227-231.

Yamada, T., S.L. Pfaff, T. Edlund, and T.M. Jessell. 1993. Control of the cell pattern in the neural tube: Motor neuron induction by diffusible factors from notochord and floor plate. Cell 73: 673-686.
Zhadanov, A., S. Bertuzzi, M. Taira, I.B. Dawid, and H. Westphal. 1995. Expression pattern of the murine LIM class homeobox gene Lhx 3 in subsets of neural and neuroendocrine tissues. Dev. Dynam. 202: 354-364. 


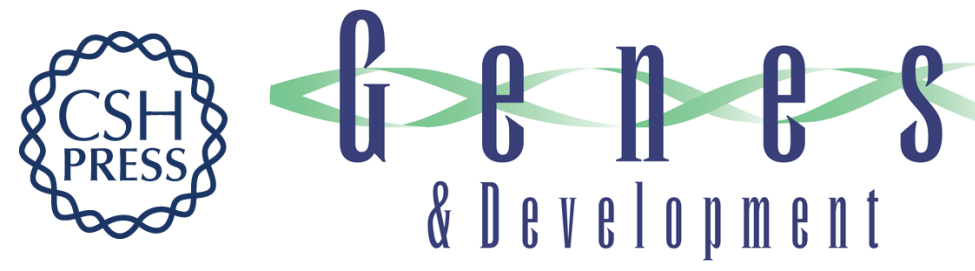

\section{A family of LIM domain-associated cofactors confer transcriptional synergism between LIM and Otx homeodomain proteins.}

I Bach, C Carrière, H P Ostendorff, et al.

Genes Dev. 1997, 11:

Access the most recent version at doi:10.1101/gad.11.11.1370

References This article cites 40 articles, 18 of which can be accessed free at:

http://genesdev.cshlp.org/content/11/11/1370.full.html\#ref-list-1

License

Email Alerting

Service

Receive free email alerts when new articles cite this article - sign up in the box at the top right corner of the article or click here.

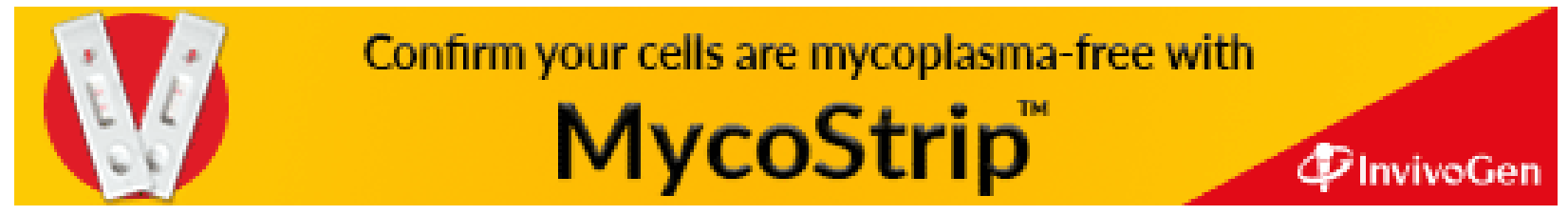

